\title{
Diretrizes normativas de conforto humano testadas em passarelas de madeira
}

\author{
Standards guidelines for human comfort tested on timber \\ footbridges
}

\author{
Pedro Gutemberg de Alcântara Segundinho ${ }^{1}$; Antonio Alves Dias² ; Marcelo \\ Rodrigo Carreira ${ }^{3}$
}

\begin{abstract}
Resumo
Atividades como caminhar, correr e pular podem produzir excitações dinâmicas indesejadas, fenômeno da ressonância devido às forças dinâmicas induzidas por pedestres, caso as passarelas apresentem frequência natural dentro das faixas de frequências de passo. A avaliação dinâmica das passarelas de madeira se faz necessária para verificar o nível de conforto humano e a sua adequação quando submetidas à passagem de pedestres. $\mathrm{O}$ objetivo deste trabalho é apresentar uma revisão bibliográfica feita nas diretrizes normativas contidas nos códigos internacionais BS 5400, SIA 160, OHBDC, CEB, EUROCODE 5, AASHTO, BRO e ISO 10137, e nos estudos de diversos pesquisadores para verificação dessas estruturas no estado limite de serviço referente às vibrações. No Brasil, as normas NBR 7190 e NBR 7188 definem os critérios para o dimensionamento das passarelas de madeira considerando os estados limites últimos e de utilização sendo que neste último não se leva em conta o conforto humano com relação a vibrações. Assim sendo, as informações contidas neste estudo podem contribuir para o aprimoramento da norma brasileira ABNT NBR 7190, pois leva em conta o conforto humano devido às vibrações induzidas por pedestres em passarelas.
\end{abstract}

Palavras-chave: Freqüências. Acelerações. Passarelas simplesmente apoiadas. Conforto humano.

\begin{abstract}
Activities such as walking, running and jumping can produce undesirable dynamic excitations, which are a phenomenon that occurs due to pedestrian-induced dynamic forces whenever a footbridge features a natural frequency within stepping frequency ranges. Dynamic evaluations of timber footbridges are necessary to verify the level of human comfort and the suitability of the bridges when subjected to human traffic. The objective of this paper is to present a bibliography review on the standards guidelines contained in international codes BS 5400, SIA 160, OHBDC, CEB, EUROCODE 5, AASHTO, BRO and ISO 10137 as well as in studies by various researchers, in order to verify the serviceability limit state for vibrations. In Brazil, codes ABNT NBR 7190 and ABNT NBR 7188 set the sizing criteria for timber footbridges considering the ultimate and serviceability limit states, with the latter ignoring human comfort with regard to vibrations. Thus, the information contained in this study can contribute to the refinement of Brazilian code ABNT NBR 7190, as it takes into account human comfort in pedestrianinduced vibrations on footbridges.
\end{abstract}

Keywords: Frequencies. Accelerations. Simply supported footbridges. Human comfort.

1 Doutor, Departamento de Engenharia de Estruturas, Escola de Engenharia de São Carlos, Universidade de São Paulo, Av. Trabalhador São-carlense, 400, 13566-590, São Carlos, SP, Brasil. E-mail: p_gutemberg2001@yahoo.com.br

2 Professor Assistente, Departamento de Engenharia de Estruturas, Escola de Engenharia de São Carlos, Universidade de São Paulo, Av. Trabalhador São-carlense, 400, 13566-590, São Carlos, SP, Brasil. E-mail: dias@sc.usp.br

3 Doutorando, Coordenação de Engenharia Civil, Universidade Tecnológica Federal do Paraná, Campus Campo Mourão, Rodovia BR 369 km 0.5, 87305-001, Campo Mourão, PR, Brasil. E-mail: carreira@utfpr.edu.br 


\section{Introdução}

Os engenheiros civis há algum tempo estão cientes dos problemas causados pelas vibrações excessivas provocadas pelo fenômeno da ressonância em passarelas. As fontes causadoras desse fenômeno são várias, podendo ter aspectos diferentes, como pedestres praticando alguns modos de caminhada, aglomerações excepcionais de pedestres, ventos fortes, chuva, passagem de veículos pesados sob elas etc. O fenômeno da ressonância ocorre devido à proximidade entre as frequências de excitação relativas às fontes e as frequências naturais da estrutura, que aumenta as amplitudes de deslocamentos, provocando desconforto. Além desse aspecto, é importante frisar que a amplitude da vibração também é influenciada pelo amortecimento da estrutura.

Em algumas situações, a atividade de caminhar, quando realizada sobre passarelas, produz vibrações excessivas que são desconfortáveis para os seres humanos e prejudiciais para essas estruturas. É importante ressaltar que o estudo do fenômeno das vibrações excessivas em passarelas causadas por pedestres não é recente no mundo. Mas, nas últimas décadas, tem ocorrido uma demanda maior desse tipo de estrutura em virtude do grande aumento da população, tanto urbana quanto rural, e assim os problemas de vibrações excessivas em passarelas têm sido mais evidentes.

As pesquisas a respeito das vibrações excessivas em passarelas já ocorrem há algum tempo. Nos últimos anos, os casos de vibrações excessivas passaram a ter maior divulgação, tornando alguns casos famosos pelo mundo, como, por exemplo, o caso da Millenium Bridge (DALLARD et al.; 2001), Londres, UK. Tal episódio deu origem a mais de 1.000 artigos e cerca de 150 programas na mídia, em todo o mundo.

O refinamento dos métodos de cálculo associado à melhoria na qualidade dos materiais estruturais resultou na produção de estruturas mais econômicas $\mathrm{e}$, consequentemente, mais esbeltas, tornando ainda mais evidente a necessidade do estudo das vibrações em estruturas.

Começou-se então a perceber uma maior suscetibilidade a vibrações excessivas dessas estruturas quando submetidas a carregamento dinâmico induzido por pedestres. As demais fontes causadoras de vibrações excessivas nas passarelas não foram levadas em conta neste trabalho, devido à sua menor ocorrência em relação ao desconforto causado aos pedestres.

As vibrações induzidas por pessoas podem conduzir as estruturas ao estado limite de serviço, pois o corpo humano é muito sensível às vibrações, (WRIGHT; GREEN, 1959; SMITH, 1988). É improvável que essas vibrações causem dano à estrutura, fato confirmado por vários casos relatados na literatura a respeito das vibrações em passarelas, nas quais, apesar de as vibrações serem excessivas, não ocorreram danos estruturais. Entretanto, deve ser reforçado que outras ações decorrentes de distúrbios ou atos de vandalismo podem causar riscos para a integridade das estruturas.

As vibrações em passarelas têm sido tratadas com atenção em vários códigos normativos de países desenvolvidos, porém existem muitas divergências na adoção dos limites para as vibrações excessivas, o que caracteriza uma indicação da incerteza de continuidade nos procedimentos de resolução dos problemas de vibrações induzidas por pedestres. Alguns códigos, como BS 5400 (1978), SIA 160 (1989), OHBDC (1991), CEB (1993), EUROCODE 5 (1993), AASHTO (1997), EUROCODE 5 (2004), BRO (2004) e ISO 10137 (2005), apresentam diretrizes detalhadas que incluem valores limites para frequências e picos de acelerações. Tais códigos possuem procedimentos de cálculo que são baseados na definição dos carregamentos de pedestres, todavia há discrepâncias entre as acelerações calculadas por meio deles e as acelerações medidas experimentalmente em passarelas. (EYRE; CULLINGTON, 1985).

O cálculo das acelerações em passarela também 
é sugerido em vários trabalhos que foram realizados nos últimos anos. Essas formulações foram desenvolvidas por Rainer, Pernica, Allen (1988), Grundmann, Kreunzinger, Schineider (1993), Pimentel e Fernandes (2002) para quantificar o valor da aceleração que os pedestres podem induzir quando caminham sobre uma passarela. Tais formulações foram feitas simplificando a estrutura real a um sistema de um grau de liberdade. As acelerações limites têm como referência as normas citadas anteriormente.

A estimativa das frequências naturais e das acelerações permite antever a natureza das vibrações excessivas e, consequentemente, avaliar o nível de conforto humano. À medida que as passarelas se tornaram mais esbeltas e leves, em decorrência do avanço tecnológico tanto em materiais como em projeto estrutural, as verificações das condições de utilização passaram a ser cada vez mais importantes. Em geral, o problema decorrente das vibrações excessivas é o desconforto causado ao usuário e também o seu receio em relação à segurança da estrutura, o que pode causar uma subutilização da passarela.

Segundo a norma International Organization for Standardization, ISO 2631-2 (1989), a percepção humana de vibrações excessivas depende muito das circunstâncias em que ocorrem: posição e atividade da pessoa, direção de incidência da vibração em relação à espinha dorsal, faixa de frequência, tempo de exposição e da expectativa que se tem em relação à vibração.

Segundo Pimentel e Fernandes (2002), as formulações atuais são, em sua maioria, aplicáveis às passarelas de sistema estrutural que podem ser modeladas como vigas retas simplesmente apoiadas. Esta modelagem simplifica o problema, pois permite incorporar os modos de vibração da estrutura à análise, já que há expressões analíticas para os mesmos. Por outro lado, as formulações simplificadas não se aplicam às passarelas de sistema estrutural estaiado ou pênsil, nas quais a interação entre os efeitos do esforço normal e a flexão produz alterações nas formas de vibrar da estrutura. Estes autores também relatam que os limites de frequências e acelerações podem ser aplicados em qualquer tipo de passarela. Entretanto, não são fáceis de serem aplicados rotineiramente, em termos de projeto, já que requerem a disponibilidade de um programa de computador que suporte a aplicação de cargas móveis variáveis deslocando-se ao longo do tempo sobre a estrutura. No sentido de tornar mais rápidas as avaliações quanto ao conforto humano, várias formulações simplificadas foram propostas e aplicadas no sistema estrutural em passarelas simplesmente apoiadas.

Dentro deste contexto, o objetivo deste trabalho é apresentar uma revisão bibliográfica feita em textos normativos e estudos de diversos pesquisadores para verificação dessas estruturas no estado limite de serviço referente às vibrações. Essas informações contribuíram para um melhor entendimento do desempenho das passarelas de madeira simplesmente apoiadas submetidas aos carregamentos dinâmicos induzidos por pedestres. O trabalho foi desenvolvido por meio de revisão bibliográfica em textos normativos e estudos de diversos autores. (SEGUNDINHO, 2010).

Em relação ao cálculo das vibrações induzidas por pedestres nas passarelas de madeira no Brasil, a norma brasileira registrada associação brasileira de normas técnicas, ABNT NBR 7190 (1997) - Projeto de Estruturas de Madeira - não fornece diretrizes para verificação desse estado limite de serviço referente aos limites de frequência e de aceleração para as passarelas de madeira, mas seu texto traz apenas as seguintes definições gerais e sucintas a respeito das recomendações de vibrações para serem atendidas nos projetos de pisos de madeira:

"Em construções submetidas a fontes de vibração, devem ser adotadas disposições construtivas que evitem a presença de vibrações excessivas da estrutura. Nas estruturas sobre as quais o público em geral pode caminhar, devem 
ser evitadas vibrações que tragam desconforto aos usuários.

No caso particular de pisos sobre os quais as pessoas andem regularmente, como os de residências e escritórios, a menor frequência natural de vibração dos elementos da estrutura do piso não deve ser inferior a $8 \mathrm{~Hz}$. Para esta finalidade, as placas compostas por elementos diagonais podem ser assimiladas a peças maciças.

Para as construções correntes, admite-se que esta condição fique satisfeita se a aplicação do carregamento correspondente à combinação de curta duração, estabelecida em 5.8.3, não provocar flecha imediata superior a $15 \mathrm{~mm}$, considerando-se o módulo de elasticidade conforme 6.4.9."

Este trabalho encontra-se organizado na seguinte sequência: critérios para avaliação do conforto humano devido às vibrações excessivas em passarelas, onde consta a formulação para o cálculo das frequências em passarelas simplesmente apoiadas; diretrizes normativas pelas quais podem ser calculadas as acelerações; considerações finais a respeito dos critérios normativos.

\section{Critérios para avaliação do conforto humano em passarelas devido às vibrações excessivas}

Cálculo das frequências naturais em passarelas simplesmente apoiadas

A determinação das frequências naturais em passarelas de madeira simplesmente apoiadas é feita a partir das expressões analíticas originadas da formulação de viga de Euler-Bernoulli. Dentre as normas estrangeiras, aquela que atualmente aborda o cálculo das frequências naturais em passarelas de madeira simplesmente apoiadas, levando-se em conta essa formulação, é o EUROCODE 5 (1993). Para essas passarelas, a primeira frequência natural na direção transversal vertical $\mathrm{f}_{1 \text {,vert }}$ pode ser calculada pela Equação 1. Para levar em conta os fatores que contribuem para a variabilidade da primeira frequência natural na direção transversal vertical $\mathrm{f}_{1, \mathrm{vert}}$, considera-se o valor mais desfavorável para o conforto do usuário entre os possíveis valores situados no intervalo de $0,7 . f_{1, \text { vert }}$ a $1,3 . f_{1, \text { vert }}$.

$$
\mathrm{f}_{1, \text { vert }}=\frac{\pi}{2 \cdot \mathrm{L}^{2}} \cdot \sqrt{\frac{\mathrm{E}_{\mathrm{c} 0 \mathrm{~m}} \cdot \mathrm{I}_{\mathrm{vert}}}{\rho_{\mathrm{ap}} \cdot \mathrm{A}_{\mathrm{sec}, \mathrm{pas}}}}[\mathrm{Hz}]
$$

Na qual:

$\rho_{a p}:$ densidade aparente da madeira $\left(\mathrm{kg} / \mathrm{m}^{3}\right)$; $\left(\mathrm{m}^{2}\right)$;

$\mathrm{A}_{\text {sec,pas }}$ : área da seção transversal da passarela

$\mathrm{E}_{\mathrm{c} 0, \mathrm{~m}}$ : módulo de elasticidade médio da madeira $\left(\mathrm{N} / \mathrm{m}^{2}\right)$;

$\mathrm{I}_{\text {vert }}$ inércia da seção da passarela de madeira na direção transversal vertical $\left(\mathrm{m}^{4}\right)$;

L: vão da passarela simplesmente apoiada ou o maior vão contínuo da passarela (m).

A primeira frequência natural na direção transversal horizontal $f_{1, \text { lat }}$ pode ser calculada pela Equação 2. Para levar em conta os fatores que contribuem para a variabilidade da frequência natural na direção transversal horizontal $\mathrm{f}_{1, \text { lat, }}$ considera-se o valor mais desfavorável para o conforto do usuário entre os possíveis valores situados no intervalo de $0,7 . f_{1, \text { lat }}$ a $1,3 . f_{1, \text { lat. }}$

$$
\mathrm{f}_{1, \text { lat }}=\frac{\pi}{2 \cdot \mathrm{L}^{2}} \cdot \sqrt{\frac{\mathrm{E}_{\mathrm{co}, \mathrm{m}} \cdot \mathrm{I}_{\mathrm{lat}}}{\rho_{\mathrm{ap}} \cdot \mathrm{A}_{\text {sec }, \text { pas }}}}[\mathrm{Hz}]
$$

Na qual:

$\rho_{a p}$ : densidade aparente da madeira $\left(\mathrm{kg} / \mathrm{m}^{3}\right)$; $\left(\mathrm{m}^{2}\right)$;

$\mathrm{A}_{\text {sec,pas }}$ : área da seção transversal da passarela

$\mathrm{E}_{\mathrm{c} 0, \mathrm{~m}}$ : módulo de elasticidade médio da madeira $\left(\mathrm{N} / \mathrm{m}^{2}\right)$;

$\mathrm{I}_{\text {lat }}$ : momento de inércia da seção transversal da passarela na direção transversal horizontal $\left(\mathrm{m}^{4}\right)$;

L: vão da passarela simplesmente apoiada ou o maior vão contínuo da passarela $(\mathrm{m})$.

Para o cálculo das frequências naturais nas 
passarelas de madeira, utilizam-se o módulo de elasticidade médio $\mathrm{E}_{\mathrm{c} 0, \mathrm{~m}}$ e o módulo de deslizamento de serviço $\mathrm{k}_{\text {ser }}$, este último quando se tratar do cálculo da rigidez de estruturas mistas, de acordo com o EUROCODE 5 (1993).

\section{Normatização a respeito de vibrações em passarelas}

Nos itens seguintes são apresentados os critérios normativos e também aqueles sugeridos por autores, para o cálculo de aceleração em passarelas. Tais critérios dizem respeito à aceitação do nível de aceleração para as passarelas em geral, isto é, não importa o sistema estrutural em que a passarela pode ser construída, uma vez que todas as passarelas serão submetidas aos mesmos critérios de aceitação. A determinação das frequências naturais e acelerações será obtida em passarelas que permitam ser modeladas como sistema estrutural de vigas retas simplesmente apoiadas.

\section{Norma BS 5400 (1978)}

A norma britânica British Standards, BS 5400 (1978) estabelece que, para estruturas cuja primeira frequência natural referente ao primeiro modo de vibrar na direção transversal vertical $f_{1, \text { vert }}$ seja abaixo de $5 \mathrm{~Hz}$, a aceleração limite na direção transversal vertical $\mathrm{a}_{\text {lim,vert }}$ em qualquer parte da estrutura deverá ser limitada ao valor calculado pela Equação 3. Caso as passarelas apresentem a primeira frequência natural referente ao primeiro modo de vibrar na direção transversal vertical $f_{1, \text { vert }}$ superior a $5 \mathrm{~Hz}$, os limites de serviço com respeito a vibrações serão atendidos.

$$
\mathrm{a}_{\text {lim, vert }}=0,5 \cdot \sqrt{\mathrm{f}_{1, \text { vert }}}\left[\mathrm{m} / \mathrm{s}^{2}\right]
$$

Na qual:

$\mathrm{f}_{1, \text { vert: }}$ primeira frequência natural da passarela na direção transversal vertical $(\mathrm{Hz})$.

Esse código apresenta um método simplificado para o cálculo do valor da aceleração na direção transversal vertical $\mathrm{a}_{\text {vert }}$, dado pela Equação 4, para passarelas simplesmente apoiadas ou contínuas de dois ou três vãos, simétricas e seção transversal constante, modeladas como vigas.

Os valores das acelerações na direção transversal vertical $a_{\text {vert }}$ obtidas por meio da Equação 4 são reduzidos de 1 a 0,7 na faixa de frequência de 4 a $5 \mathrm{~Hz}$, respectivamente, com valores intermediários sendo obtidos por meio de interpolação linear.

$$
\mathrm{a}_{\text {vert }}=4 \cdot \pi^{2} \cdot \mathrm{f}_{1, \text { vert }}^{2} \cdot \mathrm{y}_{\text {est }} \cdot \mathrm{K}_{\mathrm{BS}} \cdot \Psi\left[\mathrm{m} / \mathrm{s}^{2}\right]
$$

Na qual:

$\mathrm{f}_{1, \text { vert }}$ : primeira frequência natural da passarela na direção transversal vertical $(\mathrm{Hz})$;

$\mathrm{y}_{\text {est }}$ : flecha devido ao carregamento estático no centro do vão $(\mathrm{m}),\left(\mathrm{y}_{\text {est }}=\right.$ P.L L $^{3} / 48$.E. vert $)$, devido ao peso do pedestre $(\mathrm{P})$ igual a $700 \mathrm{~N}$;

$\mathrm{K}_{\mathrm{BS}}$ : fator de configuração que depende do número de vãos, conforme Tabela 1;

Y: fator de resposta dinâmica, obtido por meio da Figura 1. 
Tabela 1: Fator de configuração $\mathrm{K}_{\mathrm{BS}}$

\begin{tabular}{|c|c|c|}
\hline Configuração da passarela & $\mathrm{L}_{1} / \mathrm{L}$ & $\mathrm{K}_{\mathrm{Bs}}$ \\
\hline$\triangle \quad \mathrm{L} \quad \triangle$ & - & 1,0 \\
\hline$\triangle \quad$ L $\triangle \quad$ L $\triangle$ & - & 0,7 \\
\hline$\triangle \quad \mathrm{L}_{1} \quad \triangle \quad \mathrm{L} \quad \triangle \quad \mathrm{L}_{1} \triangle$ & $\begin{array}{l}1,0 \\
0,8 \\
\leq 0,6\end{array}$ & $\begin{array}{l}0,6 \\
0,8 \\
0,9\end{array}$ \\
\hline
\end{tabular}

Figura 1: Fator de resposta dinâmica em função do comprimento do vão e da taxa de amortecimento da passarela

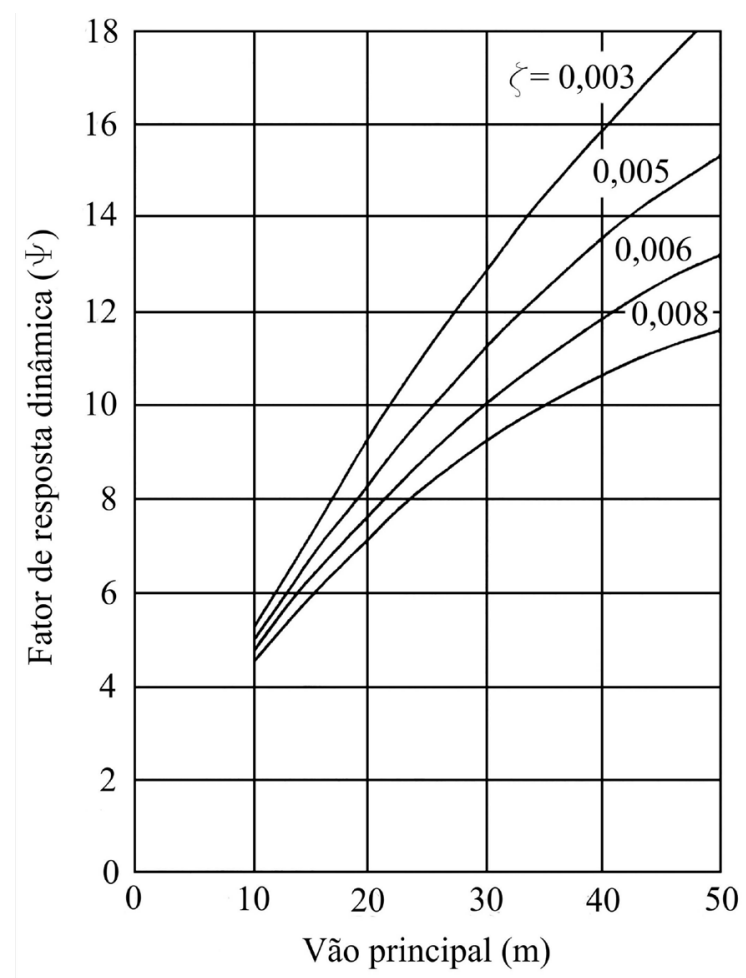

Nas estruturas mais complexas que apresentam formas diferentes daquelas apresentadas na Tabela 1, o valor da aceleração na direção transversal vertical $a_{\text {vert }}$ pode ser calculado assumindo que o carregamento dinâmico aplicado por um pedestre é representado por uma carga $\mathrm{F}(\mathrm{t})$, Equação 5, pulsando harmonicamente ao longo da passarela com uma velocidade constante na direção transversal vertical $v_{\text {vert }}$, dada pela Equação 6 . 


$$
\begin{aligned}
& F(t)=180 \cdot \operatorname{sen}\left(2 \cdot \pi \cdot f_{1, \text { vert }} \cdot t\right)[N] \\
& v_{\text {vert }}=0,9 \cdot f_{1, \text { vert }}[\mathrm{m} / \mathrm{s}]
\end{aligned}
$$

Na qual:

$\mathrm{f}_{1, \text { vert }}$ : primeira frequência natural da passarela na direção transversal vertical $(\mathrm{Hz})$;

t: tempo de duração do carregamento (s).

A desvantagem da utilização das Equações 4 e 5 é que foi adotado, para as mesmas, o primeiro fator dinâmico de carga $(\alpha 1)$ com valor igual a 0,257 , sugerido por Blanchard, Davies, Smith (1977), referente ao primeiro harmônico para toda a faixa de frequências abaixo de $5 \mathrm{~Hz}$, não se levando em consideração os fatores dinâmicos de carga correspondentes aos harmônicos superiores que podem ocorrer dentro dessa faixa de frequência. $\mathrm{Na}$ Equação 4, este fator dinâmico de carga está incluso na obtenção do gráfico do fator de resposta dinâmica (Y), no qual também não é levado em conta o número de ciclos (n) que está relacionado com a frequência de passo $\left(f_{p}\right)$, ou seja, a passarela pode ser excitada pelo primeiro ou pelo segundo harmônico. Neste segundo caso, o número de ciclos será o dobro da frequência de passo $\left(f_{p}\right)$. Na Equação 5, o fator dinâmico de carga $\left(\alpha_{1}\right)$ igual a 0,257, sugerido por Blanchard, Davies, Smith (1977), está incluso na amplitude do primeiro harmônico do carregamento de caminhada $(180 \mathrm{~N}$ $=0,257.700 \mathrm{~N})$, sendo o peso do pedestre $(\mathrm{P})$ igual a 700 N. O fator de configuração $K_{B S}$ corrige o valor da aceleração na direção transversal vertical $a_{\text {vert }}$ dada pela Equação 4, ao aplicar-se para passarelas de até três vão contínuos, expandindo a mesma.

\section{Norma OHBDC (1991)}

A norma canadense OHBDC (1991) apresenta diferenças em relação à norma BS 5400 (1978). A aceleração limite na direção transversal vertical $\mathrm{a}_{\text {lim,vert }}$ será verificada caso a primeira frequência natural na direção transversal vertical $f_{1, \text { vert }}$ seja menor que $4 \mathrm{~Hz}$. Esta norma adota um valor mais conservador para a aceleração limite na direção transversal vertical $a_{\text {lim,vert }}$ calculada por meio da Equação 7.

$$
\mathrm{a}_{\text {lim, vert }}=0,25 \cdot \mathrm{f}_{1, \text { vert }} 0,78\left[\mathrm{~m} / \mathrm{s}^{2}\right]
$$

Na qual:

$\mathrm{f}_{1 \text {,vert }}$ : primeira frequência natural da passarela na direção transversal vertical (Hz).

O valor da aceleração na direção transversal vertical avert da norma canadense OHBDC (1991) é calculado por meio do método simplificado da norma britânica BS 5400 (1978), utilizando a Equação 4. O mesmo ocorre quando se tratar de estruturas mais complexas que apresentam formas diferentes daquelas mostradas na Tabela 1, na qual o valor da aceleração na direção transversal vertical avert será calculado pela Equação 5 .

\section{Norma EUROCODE 5 (1993) - EC5}

A norma europeia EUROCODE 5 (1993) recomenda que sejam dispensadas das verificações de acelerações limites, na direção transversal vertical e transversal horizontal, as passarelas que apresentam as primeiras frequências naturais na direção transversal vertical e transversal horizontal acima de $5 \mathrm{Hze} 2,5 \mathrm{~Hz}$, respectivamente. Essa norma contém diretrizes para o projeto de passarelas de madeira, referentes à determinação das acelerações na direção transversal vertical e transversal horizontal para um pequeno grupo de pessoas, cujas primeiras frequências naturais estejam abaixo 
dos limites estabelecidos. O documento estabelece ainda um valor de aceleração limite na direção transversal vertical $\mathrm{a}_{\text {lim,vert }}$ menor ou igual a $0,7 \mathrm{~m} / \mathrm{s}^{2}$ ( $7 \%$ g). O cálculo do valor da aceleração na direção transversal vertical $a_{\text {vert }}$ para passarelas de madeira com a primeira frequência natural na direção transversal vertical $f_{1, \text { vert }}$ não excedendo $5 \mathrm{~Hz}$, que possuem um, dois ou três vãos, conforme mostrado na Tabela 2, é realizado por meio da Equação 8. Esta permite o cálculo do valor da aceleração na direção transversal vertical $a_{\text {vert }}$ para um pequeno fluxo de pessoas, que é uma situação normal para a maior parte das passarelas de madeira.

$$
\mathrm{a}_{\text {vert }}=\mathrm{a}_{1, \text { vert }} \cdot \mathrm{k}_{\mathrm{vert}, \mathrm{f}}\left[\mathrm{m} / \mathrm{s}^{2}\right]
$$

Na qual:

$\mathrm{k}_{\text {vert,ff }}$ fator de grupo relacionado à frequência natural da passarela, ver Figura 2.
O cálculo do valor da aceleração na direção transversal vertical $\mathrm{a}_{1, \mathrm{vert}}$, sem levar em conta o fator de grupo $\mathrm{k}_{\mathrm{vert}, \mathrm{f}}$ é realizado por meio da Equação 9:

$$
\begin{aligned}
& \mathrm{a}_{1, \text { vert }}=165 \cdot \mathrm{k}_{\mathrm{a}} \cdot \frac{1-\mathrm{e}^{-2 \cdot \pi \cdot n \cdot \zeta}}{\mathrm{M} \cdot \zeta}\left[\mathrm{m} / \mathrm{s}^{2}\right] \\
& \mathrm{M}=\rho_{\text {ap }} \cdot \mathrm{A}_{\text {sec, pas }} \cdot \mathrm{L}
\end{aligned}
$$

Na qual:

M: massa total da passarela $(\mathrm{kg})$;

$\rho_{a p}:$ densidade aparente da madeira $\left(\mathrm{kg} / \mathrm{m}^{3}\right)$; $\left(\mathrm{m}^{2}\right)$;

$\mathrm{A}_{\text {sec,pas }}$ : área da seção transversal da passarela

$\mathrm{L}$ : vão da passarela $(\mathrm{m})$;

$\zeta:$ taxa de amortecimento;

n: número de passos para cruzar o vão da passarela $\mathrm{L}(\mathrm{m}),(n=\mathrm{L} / 0,9 \mathrm{~m})$;

ka: fator de configuração, conforme Tabela 2.

Tabela 2: Fator de configuração ka

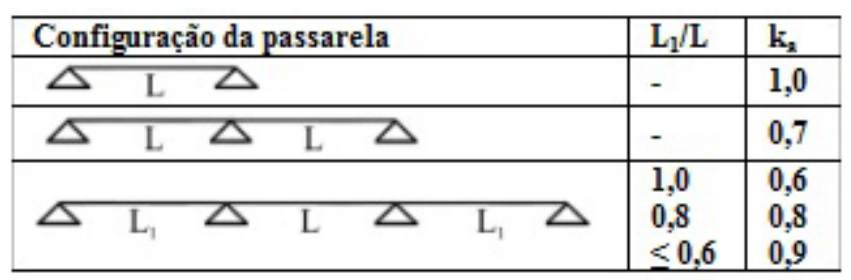


Figura 2: Relação entre o fator de grupo $\mathrm{k}_{\text {vert,f }}$ e a primeira frequência natural da passarela na direção transversal vertical $f_{1, \text { vert }}$

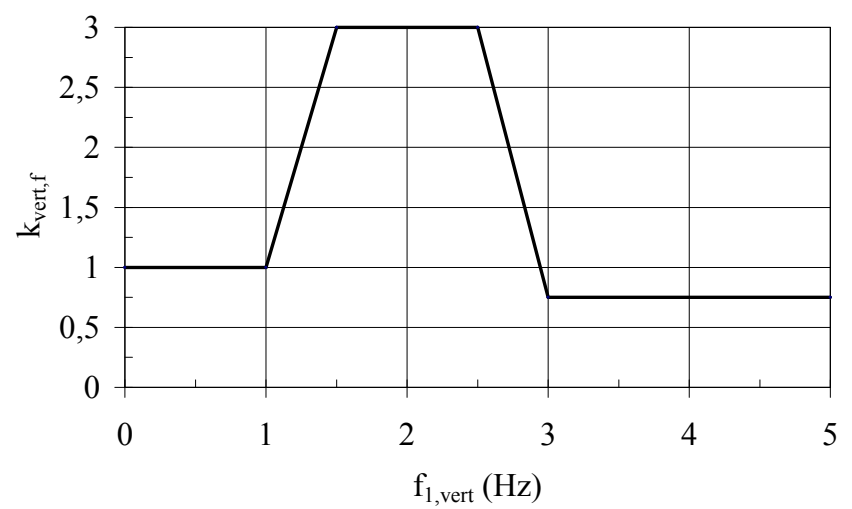

O cálculo do valor da aceleração na direção transversal vertical $a_{\text {vert }}$ devido a um pedestre cruzando uma passarela é realizado por meio da Equação 11:

$$
a_{\text {vert }}=0,027 \cdot \mathrm{L} \cdot \mathrm{b} \cdot \mathrm{a}_{1, \text { vert }} \cdot \mathrm{k}_{\text {vert,f }}\left[\mathrm{m} / \mathrm{s}^{2}\right]
$$

Na qual:

L: vão da passarela (m);

b: largura da passarela (m).

Para configurações de passarelas diferentes das apresentadas na Tabela 2, o valor da aceleração na direção transversal vertical $a_{\text {vert }}$ pode ser obtido a partir da força aplicada na direção transversal vertical, Equação 12, exercida por um pedestre movimentando-se ao longo da passarela com velocidade na direção transversal vertical $\mathrm{v}_{\text {vert }}$, obtida pela Equação 13:

$$
\begin{aligned}
& \mathrm{F}(\mathrm{t})=0,28 \cdot \operatorname{sen}\left(2 \cdot \pi \cdot \mathrm{f}_{1, \text { vert }} \cdot \mathrm{t}\right)[\mathrm{kN}] \\
& \mathrm{v}_{\text {vert }}=0,9 \cdot \mathrm{f}_{1, \text { vert }}[\mathrm{m} / \mathrm{s}]
\end{aligned}
$$

Na qual:

$\mathrm{f}_{1, \text { vert }}$ : primeira frequência natural da passarela na direção transversal vertical $(\mathrm{Hz})$;

t: tempo de duração do carregamento (s).

Este código normativo apresenta uma expressão simplificada, Equação 14, para a determinação do valor da aceleração na direção transversal horizontal $\mathrm{a}_{\text {lat }}$ para passarelas de madeira com frequência natural não excedendo $2,5 \mathrm{~Hz}$, que possuem um, dois ou três vãos, conforme mostrado na Tabela 2. Esta norma estabelece uma aceleração limite na direção transversal horizontal $a_{\text {lim,lat }}$ menor ou igual a 0,2 $\mathrm{m} / \mathrm{s}^{2}(2 \% \mathrm{~g})$. A Equação 14 considera o cálculo do valor da aceleração na direção transversal horizontal $\mathrm{a}_{\text {lat }}$ para um pequeno fluxo de pessoas, que é uma situação normal para a maior parte das passarelas de madeira.

$$
\mathrm{a}_{\mathrm{lat}}=\mathrm{a}_{1, \mathrm{lat}} \cdot \mathrm{k}_{\mathrm{lat}, \mathrm{f}}\left[\mathrm{m} / \mathrm{s}^{2}\right]
$$

Na qual:

$\mathrm{k}_{\text {lat,f }}$ : fator de grupo relacionado à frequência natural da passarela; ver Figura 3.

O cálculo do valor da aceleração transversal horizontal $\mathrm{a}_{1, \text { lat }}$, sem levar em conta o fator de grupo 
$\mathrm{k}_{\text {lat,f }}$, é realizado por meio da Equação 15:

$$
\begin{aligned}
& \mathrm{a}_{1, \text { lat }}=40 \cdot \mathrm{k}_{\mathrm{a}} \cdot \frac{1-\mathrm{e}^{-2 \cdot \pi \cdot n \cdot \zeta}}{\mathrm{M} \cdot \zeta}\left[\mathrm{m} / \mathrm{s}^{2}\right] \\
& \mathrm{M}=\rho_{\text {ap }} \cdot \mathrm{A}_{\text {sec,pas }} \cdot \mathrm{L}
\end{aligned}
$$

Na qual:

M: massa total da passarela $(\mathrm{kg})$;

$\rho_{a p}:$ densidade aparente da madeira $\left(\mathrm{kg} / \mathrm{m}^{3}\right)$; $\left(\mathrm{m}^{2}\right)$;

$\mathrm{A}_{\text {sec,pas }}$ : área da seção transversal da passarela

L: vão da passarela (m);

$\zeta:$ taxa de amortecimento;

$n$ : número de passos para cruzar o vão da passarela $\mathrm{L}(\mathrm{m}),(n=\mathrm{L} / 0,9)$;

$\mathrm{k}_{\mathrm{a}}$ : fator de configuração, conforme Tabela 2.

Figura 3: Relação entre o fator de grupo $\mathrm{k}_{\text {latff }}$ e a primeira frequência natural da passarela na direção transversal horizontal $\mathrm{f}_{1, \text { lat }}$

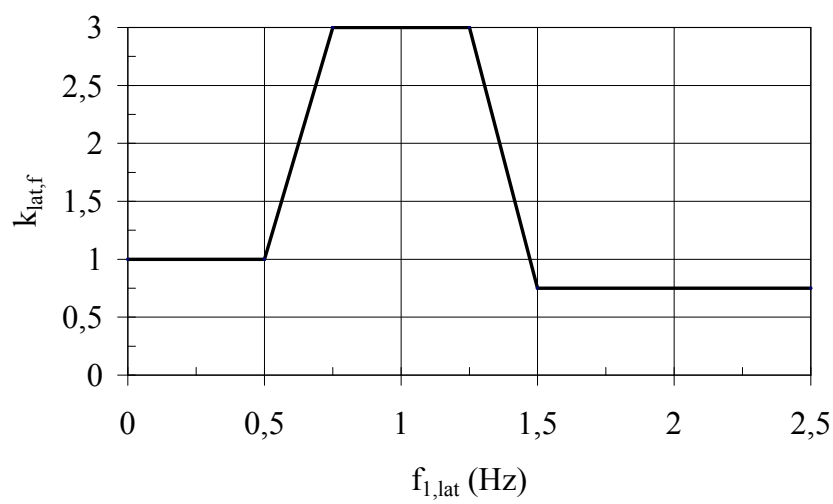

O cálculo do valor da aceleração na direção transversal horizontal $\mathrm{a}_{\text {lat }}$ devido a um pedestre cruzando uma passarela é realizado por meio da Equação 17:

$$
\mathrm{a}_{\text {lat }}=0,027 \cdot \mathrm{L} \cdot \mathrm{b} \cdot \mathrm{a}_{1, \text { lat }} \cdot \mathrm{k}_{\text {lat }, \mathrm{f}}\left[\mathrm{m} / \mathrm{s}^{2}\right]
$$

Na qual:

$$
\begin{aligned}
& \text { L: vão da passarela (m); } \\
& \text { b: largura da passarela (m). }
\end{aligned}
$$

Para configurações de passarelas diferentes das apresentadas na Tabela 2, o valor da aceleração na direção transversal horizontal $\mathrm{a}_{\text {lat }}$ pode ser calculado a partir da força aplicada na direção transversal horizontal, Equação 18, exercida por um pedestre movimentando-se ao longo da passarela, com velocidade na direção transversal horizontal $\mathrm{v}_{\text {lat }}$, obtida pela Equação 19:

$$
\begin{aligned}
& \mathrm{F}_{\text {lat }}(\mathrm{t})=0,07 \cdot \operatorname{sen}\left(2 \cdot \pi \cdot \mathrm{f}_{1, \text { lat }} \cdot \mathrm{t}\right)[\mathrm{kN}] \\
& \mathrm{v}_{\text {lat }}=1,8 \cdot \mathrm{f}_{1, \text { lat }}[\mathrm{m} / \mathrm{s}]
\end{aligned}
$$

Na qual:

$\mathrm{f}_{1, \text { lat }}$ : primeira frequência natural da passarela na direção transversal horizontal $(\mathrm{Hz})$;

t: tempo de duração do carregamento (s).

A norma europeia EUROCODE 5 (1993) traz, em seu escopo, as seguintes observações: $\mathrm{O}$ 
método simplificado de cálculo apresentado pela norma europeia EUROCODE 5 (1993) supõe que a frequência de caminhada dos pedestres seja correspondente à primeira frequência natural da estrutura ou, então, seja a metade desta; na falta de valores precisos, recomenda-se adotar o valor de taxa de amortecimento $\zeta$ igual a 0,010, para estruturas sem ligações e 0,015 , para estruturas com ligações; as Equações 8 e 14 são relevantes quando se tratar de pequenas passarelas que possuam área de piso menor ou igual a $37 \mathrm{~m}^{2}$. Os fatores de grupo $\mathrm{k}_{\mathrm{vert}, \mathrm{f}}$ e $\mathrm{k}_{\mathrm{lat}, \mathrm{f}}$ são dependentes da primeira frequência natural da passarela para as direções transversal vertical ou transversal horizontal e referem-se à ação de um grupo de 10 pedestres ou à passagem de pedestres que representem uma densidade de 0,6 pessoas $/ \mathrm{m}^{2}$.

Normas SIA 160 (1989), CEB (1993) e AASHTO (1997)

As normas SIA 160 (1989), CEB (1993) e AASHTO (1997) abordam, por exclusão, o problema de vibrações verticais nas passarelas. As duas primeiras normas reconhecem as faixas críticas de frequências produzidas por um pedestre, ao caminhar, e recomendam que seja evitado projetar passarelas com frequências naturais na direção transversal vertical entre 1,6 e $2,4 \mathrm{~Hz}$ e também entre 3,5 e 4,5 Hz. Tais limitações são baseadas no fato de que a frequência de caminhada está distribuída dentro de uma gama de 1,6 a 2,4 Hz e 3,2 a $4,8 \mathrm{~Hz}$, que são as faixas do primeiro e segundo harmônicos, respectivamente, segundo Pimentel, Pavic, Waldron (2001). A AASHTO (1997) também reconhece a mesma abordagem e recomenda que seja evitado projetar passarelas com frequências naturais na direção transversal vertical inferiores a $3 \mathrm{~Hz}$ e $5 \mathrm{~Hz}$, aplicando o segundo limite, caso sejam esperados problemas de vibração causados pelo segundo harmônico do movimento de caminhada.

\section{Norma BRO (2004)}

BRO (2004) é uma norma aplicada para os projetos e construções das pontes na Suécia, publicada pela SRA (Swedish Road Administration). A SRAé a autoridade nacional nomeada responsável por vários setores para assegurar o transporte no sistema de estradas. A SRA também é responsável pelo planejamento, construção, operação e manutenção do estado das rodovias.

A BRO (2004) declara que as passarelas devem ter frequência fundamental de vibração na direção transversal vertical acima de $3,5 \mathrm{~Hz}$. Consequentemente, a passarela deve ser verificada para as vibrações de serviço. Caso uma frequência natural de vibração na direção transversal vertical seja menor ou igual a $3,5 \mathrm{~Hz}$, verifica-se que a aceleração limite na direção transversal vertical $\left(\mathrm{a}_{\text {lim,RMS }}=\mathrm{a}_{\text {vert }} / 2^{0,5}\right)$ para qualquer parte da passarela deve ser menor ou igual a $0,5 \mathrm{~m} / \mathrm{s}^{2}$. O valor da aceleração na direção transversal vertical $\mathrm{a}_{\text {vert }}$ é obtido a partir da análise dinâmica que pode ser realizada por meio de qualquer um dos métodos simplificados ou um método geral. O método simplificado dado nesta norma é aplicado somente para passarelas simplesmente apoiadas. Para estruturas mais complexas, é recomendada uma análise detalhada usando manuais ou programas de computadores.

O valor da aceleração na direção transversal vertical deve ser calculado assumindo que o carregamento dinâmico aplicado por um pedestre é representado por uma carga estacionária vibrando, dada pela Equação 20. Esta aborda somente a aceleração limite na direção transversal vertical $a_{\text {lim,vert }}$ e não exige ou considera precauções de vibrações na direção transversal horizontal que sejam estabelecidas fora do código.

$$
F(t)=k_{1} \cdot k_{2} \cdot \operatorname{sen}\left(2 \cdot \pi \cdot f_{p} \cdot t\right)[N]
$$


Na qual:

$\mathrm{k}_{1}$ : valor obtido por $\mathrm{k}_{1}=(0,1 . \mathrm{b} . \mathrm{L})^{0,5} ; \mathrm{b}$ e $\mathrm{L}$ são, respectivamente, largura e vão da passarela;

$\mathrm{k}_{2}$ : carregamento constante $\left(\mathrm{k}_{2}=150 \mathrm{~N}\right)$;

$\mathrm{f}_{\mathrm{p}}$ : frequência de passo $(\mathrm{Hz})$;

t: tempo de duração do carregamento (s).

\section{Norma ISO 10137 (2005)}

A norma ISO 10137 (2005) foi desenvolvida com o objetivo de apresentar os princípios para prever vibrações na fase de projeto e avaliar a aceitabilidade de vibrações em estruturas. Esta define fonte, caminho e receptor de vibração como três assuntos-chaves que requerem consideração ao lidar com as vibrações da estrutura no estado limite de serviço. Os pedestres são fontes de vibração que produzem forças ou ações dinâmicas na estrutura.
De acordo com a ISO 10137 (2005), a análise de resposta requer um cálculo padrão que incorpore as características da fonte e do caminho de transmissão, que é então resolvido para a resposta de vibração no receptor.

Nesta norma, o projetista decidirá com qual critério de estado limite de serviço serão projetadas as passarelas, cujas fontes de amplitudes de vibração não alarmem os potenciais usuários. É sugerido usar as curvas básicas para vibrações nas direções transversal vertical e transversal horizontal dadas na ISO 2631-2 (1989), Figuras 4 e 5, porém as curvas de aceitabilidade propostas pela norma ISO 2631-2 (1989) não estão baseadas em pesquisas pertinentes a vibrações que ocorrem nas passarelas, segundo Zivanovic, Pavic, Reynolds (2005). A variação de aceleração nessas curvas difere das propostas anteriores, uma vez que é dada em RMS (rootmean-square).

Figura 4: Curva base de frequência versus aceleração na direção transversal vertical.

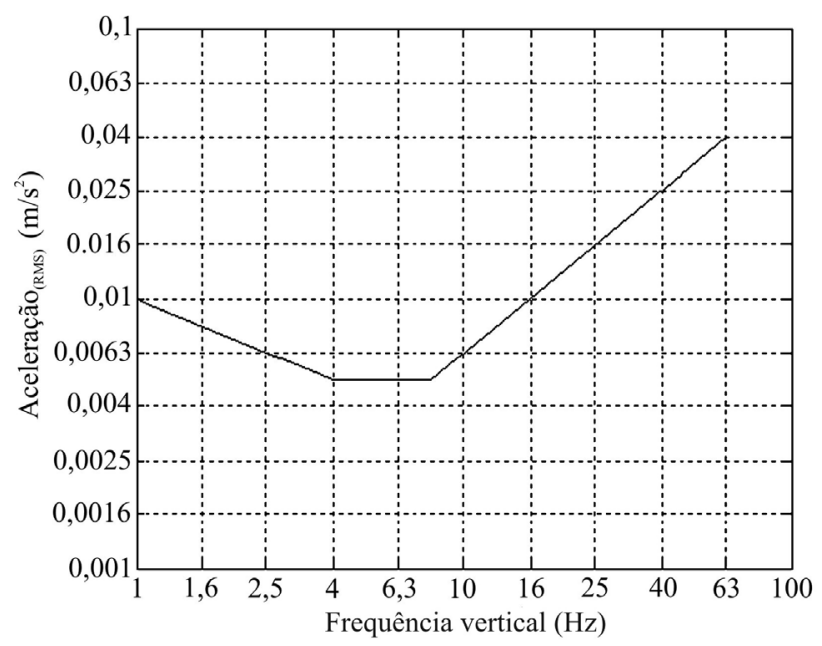


Figura 5: Curva base de frequência versus aceleração na direção transversal horizontal.

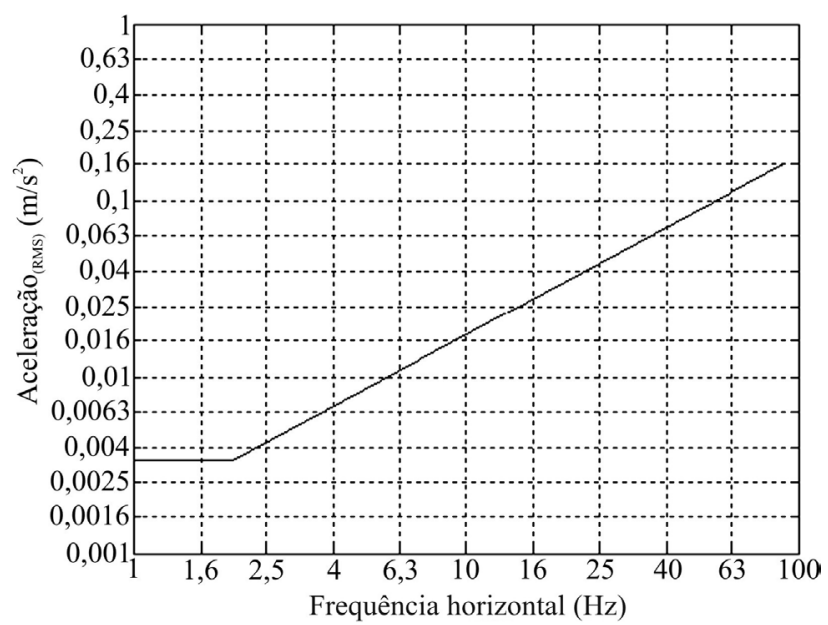

De acordo com a ISO 10137 (2005), as ações dinâmicas de uma ou mais pessoas podem ser apresentadas como força no tempo, pois variam no tempo e espaço ao longo da estrutura. A situação do projeto deve ser selecionada dependendo do tráfego a ser admitido na passarela durante a vida útil. Ainda segundo esta norma, a força dinâmica $F(t)$ produzida por um pedestre caminhando ao longo de uma passarela pode ser expressa no domínio do tempo, como uma série de Fourier, Equações 21 e 22:

$\mathrm{F}_{\text {vert }}(\mathrm{t})=\mathrm{P}\left[1+\sum_{\mathrm{i}=1}^{\mathrm{N}} \alpha_{\mathrm{i}, \text { vert }} \cdot \operatorname{sen}\left(2 \cdot \pi \cdot \mathrm{i} \cdot \mathrm{f}_{\mathrm{p}} \cdot \mathrm{t}-\varphi_{\mathrm{i}, \text { vert }}\right)\right][\mathrm{N}]$

$\mathrm{F}_{\text {lat }}(\mathrm{t})=\mathrm{P}\left[1+\sum_{\mathrm{i}=1}^{\mathrm{N}} \alpha_{\mathrm{i}, \text { lat }} \cdot \operatorname{sen}\left(2 \cdot \pi \cdot \mathrm{i} \cdot \mathrm{f}_{\mathrm{p}} \cdot \mathrm{t}-\varphi_{\mathrm{i}, \text { lat }}\right)\right][\mathrm{N}]$

Na qual:

P: peso do pedestre igual a $700 \mathrm{~N}$;

$\alpha_{i}$ : coeficiente de Fourier do ith harmônico; este corresponde ao fator de carregamento dinâmico na direção transversal vertical ou transversal horizontal, Tabelas 5 e 6 ;

$\mathrm{f}_{\mathrm{p}}$ : frequência de passo $(\mathrm{Hz})$;

$\varphi_{\mathrm{i}}$ : ângulo de fase para o ith harmônico relacionado à direção transversal vertical ou transversal horizontal, Tabelas 5 e 6 ;

i: número do ith harmônico;

$\mathrm{N}$ : número total de harmônicos;

t: tempo de duração do carregamento (s).

\section{Norma EUROCODE 5 (2004) - EC5*}

A norma europeia EUROCODE 5 (2004) permite que sejam dispensadas das verificações de acelerações as passarelas que apresentarem frequências naturais nas direções transversal vertical e transversal horizontal acima de 5,0 Hz e 2,5 Hz, respectivamente. Este código propõe, nesta versão de avaliação, apenas diretrizes para projeto de passarelas de madeira simplesmente apoiadas de um único vão, sem levar em conta os casos de passarelas de madeira contínuas de dois e três vãos. A seguir, tem-se o cálculo das acelerações nas direções transversal vertical e transversal horizontal, quando uma pessoa ou várias pessoas cruzam uma passarela de madeira, cujas frequências naturais da estrutura estejam abaixo dos limites estabelecidos. $\mathrm{O}$ cálculo da aceleração na direção transversal vertical $a_{1, \text { vert }}$ para uma pessoa cruzando, em movimento de caminhada, uma passarela de madeira simplesmente apoiada é dado pela Equação 23: 


$$
a_{1, \text { vert }}=\left\{\begin{array}{l}
\frac{200}{M \cdot \zeta} \text { para }_{1, \text { vert }} \leq 2,5 \mathrm{~Hz}\left[\mathrm{~m} / \mathrm{s}^{2}\right] \\
\frac{100}{M \cdot \zeta} \text { para } 2,5 \mathrm{~Hz}<\mathrm{f}_{1, \text { vert }} \leq 5,0 \mathrm{~Hz}\left[\mathrm{~m} / \mathrm{s}^{2}\right]
\end{array}\right.
$$

O cálculo da aceleração na direção transversal vertical $a_{\text {np,vert }}$ para vários pedestres cruzando, em movimento de caminhada, uma passarela de madeira é dado pela Equação 24:

$$
\mathrm{a}_{\mathrm{np}, \text { vert }}=0,23 \cdot \mathrm{a}_{1, \text { vert }} \cdot n p \cdot \mathrm{k}_{1, \text { vert }}\left[\mathrm{m} / \mathrm{s}^{2}\right]
$$

O cálculo da aceleração na direção transversal vertical $a_{1, \text { vert }}$ para um pedestre cruzando, em movimento de corrida, uma passarela de madeira é dado pela Equação 25:

$$
a_{1, \text { vert }}=\frac{600}{M \cdot \zeta} \text { para } 2,5<\mathrm{f}_{1, \text { vert }} \leq 3,5 \mathrm{~Hz}\left[\mathrm{~m} / \mathrm{s}^{2}\right]
$$

O cálculo da aceleração na direção transversal horizontal $\mathrm{a}_{1, \text { lat }}$ para uma pessoa cruzando, em movimento de caminhada, uma passarela de madeira é dado pela Equação 26:

$$
\mathrm{a}_{1, \text { lat }}=\frac{50}{\mathrm{M} \cdot \zeta} \text { para } 0,5 \mathrm{~Hz} \leq \mathrm{f}_{1, \text { lat }} \leq 2,5 \mathrm{~Hz}\left[\mathrm{~m} / \mathrm{s}^{2}\right]
$$

O cálculo da aceleração na direção transversal horizontal $\mathrm{a}_{\mathrm{np}, \mathrm{lat}}$ para vários pedestres cruzando, em movimento de caminhada, uma passarela de madeira é dado pela Equação 27:

$$
\mathrm{a}_{\mathrm{np}, \text { lat }}=0,18 \cdot \mathrm{a}_{1, \text { lat }} \cdot n p \cdot \mathrm{k}_{1, \text { lat }}\left[\mathrm{m} / \mathrm{s}^{2}\right]
$$

Na qual:

$n p$ : número de pedestres;

$\mathrm{M}$ : massa total da passarela $(\mathrm{kg})$ é igual a $\mathrm{M}=\rho_{a p}$. $\mathrm{A}_{\text {sec,pas }} . \mathrm{L}$;

L: vão da passarela $(\mathrm{m})$;

b: largura da passarela (m);

$\rho_{a p}:$ densidade aparente da madeira $\left(\mathrm{kg} / \mathrm{m}^{3}\right)$;

$\mathrm{A}_{\text {sec }}$ pas: área da seção transversal da passarela $\left(\mathrm{m}^{2}\right)$;

$\zeta$ : taxa de amortecimento;

$\mathrm{k}_{1, \mathrm{ver}} \mathrm{t}$ : coeficiente relacionado à primeira frequência natural da passarela de madeira na direção transversal vertical $f_{1, \text { vert }}$, Figura 6;

$\mathrm{k}_{1, \text { lat }}$ : coeficiente relacionado à primeira frequência natural da passarela de madeira na direção transversal horizontal f1,lat, Figura 7.

Figura 6: Coeficiente $k_{1, \text { vert }}$ relacionado à primeira frequência natural na direção transversal vertical $f_{1, \text { vert }}$ da passarela de madeira

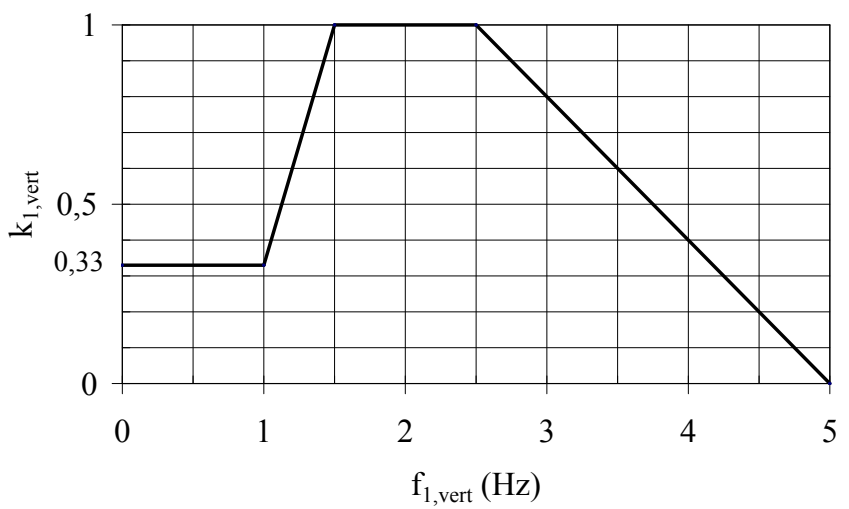


Figura 7: Coeficiente $\mathrm{k}_{1, \text { lat }}$ relacionado à primeira frequência natural na direção transversal horizontal $\mathrm{f}_{1, \text { lat }}$ da passarela de madeira.

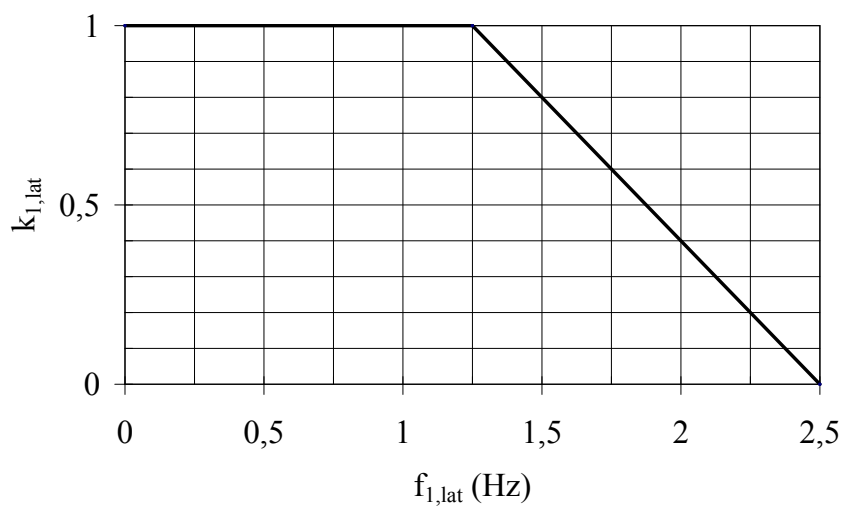

\section{Notas:}

$\mathrm{Na}$ falta de valores precisos, podem ser adotados valores de coeficiente de amortecimento $\zeta$ iguais a 0,010, para estruturas sem ligações, e 0,015, para estruturas com ligações;

O número de pedestres $n p$ é considerado igual a 13, quando se toma um grupo distinto de pedestres, e igual a $6 . \mathrm{A}_{\mathrm{p}}$, quando se tem um grupo contínuo de pedestres, sendo Ap a área em $\left(\mathrm{m}^{2}\right)$ do piso da passarela de madeira $\left(A_{p}=b . L\right)$;

O EUROCODE 5 (2004) mantém as acelerações limites nas direções transversal vertical alim,vert menor ou igual a $0,7 \mathrm{~m} / \mathrm{s}^{2}(7 \% \mathrm{~g})$ e transversal horizontal alim,lat menor ou igual a $0,2 \mathrm{~m} / \mathrm{s}^{2}(2 \%$ g).

Trabalhos relacionados às acelerações em passarelas

O cálculo das acelerações em passarela é sugerido em vários trabalhos que foram realizados nos últimos anos. Essas formulações, desenvolvidas para quantificar o valor da aceleração que os pedestres podem induzir quando caminham sobre uma passarela, foram feitas por meio de uma simplificação da estrutura real, adotando sistema de um grau de liberdade. As acelerações limites têm como referência as normas citadas anteriormente.

Rainer, Pernica, Allen (1988) propuseram um método para o cálculo da aceleração na direção transversal vertical que resulta da passagem de pedestre caminhando ou correndo com frequência ressonante na passarela, Equação 28, realizaram a modelagem para oscilações de sistema de um grau de liberdade e sugeriram que as frequências e acelerações não ultrapassem os limites estabelecidos em normas.

$$
\mathrm{a}_{\text {vert }}=4 \cdot \pi^{2} \cdot \mathrm{f}_{1, \text { vert }}^{2} \cdot \mathrm{y}_{\text {est }} \cdot \alpha_{\mathrm{i}} \cdot \Phi\left[\mathrm{m} / \mathrm{s}^{2}\right]
$$

Na qual:

$\mathrm{f}_{1 \text {,vert }}$ : primeira frequência natural da passarela na direção transversal vertical $(\mathrm{Hz})$;

$\mathrm{y}_{\text {est }}$ : flecha devida ao carregamento estático no centro do vão $(\mathrm{m}),\left(\mathrm{y}_{\text {est }}=\right.$ P.L $\mathrm{L}^{3} / 48$.E. I $\left._{\text {vert }}\right)$, devido ao peso do pedestre $(\mathrm{P})$ igual a $700 \mathrm{~N}$;

$\alpha_{i}$ : coeficiente de Fourier, Tabela 5. Este coeficiente foi obtido a partir de ensaios experimentais realizados em plataformas rígidas sob ação de pessoas caminhando;

$\Phi$ : fator de amplificação dinâmico obtido graficamente em Rainer, Pernica, Allen (1988), de acordo com a Figura 8, a partir do número de ciclos por vão ( $\mathrm{n}^{\mathrm{o}} \mathrm{i}$ th harmônico.L/1 $\mathrm{p}$ ), do comprimento de passo $\left(1_{\mathrm{p}}\right)$ dado na Tabela 3 , necessário para os pedestres cruzarem uma passarela simplesmente apoiada com taxa de amortecimento $\zeta$. 
Tabela 3: Valores típicos para frequência de passo $\left(f_{p}\right)$, velocidade $\left(v_{p}\right)$ e comprimento $\left(l_{p}\right)$. Fonte: (BACHMANN; AMMANN, 1987)

\begin{tabular}{llll}
\hline & $\mathbf{f}_{\mathbf{p}}(\mathbf{H z})$ & $\mathbf{v}_{\mathbf{p}}(\mathbf{m} / \mathbf{s})$ & $\mathbf{l}_{\mathbf{p}}(\mathbf{m})$ \\
\hline Caminhada lenta & 1,7 & 1,0 & 0,60 \\
Caminhada normal & 2,0 & 1,5 & 0,75 \\
Caminhada rápida & 2,3 & 2,3 & 1,00 \\
Corrida normal & 2,5 & 3,1 & 1,25 \\
Corrida rápida & $>3,2$ & 5,5 & 1,75 \\
\hline
\end{tabular}

Figura 8: Fator de amplificação dinâmica para resposta ressonante devido à carga móvel senoidal cruzando um vão simples. Fonte: (RAINER, PERNICA, ALLEN, 1988)

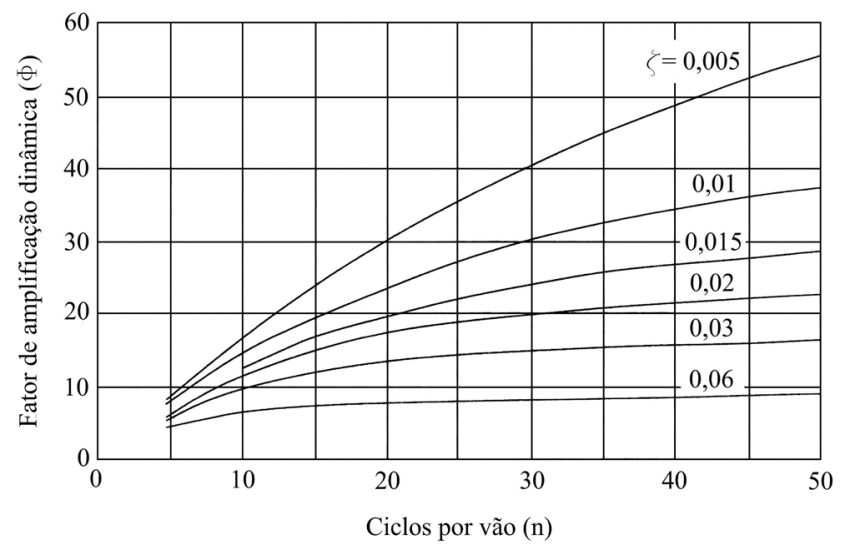

A Equação 28 pode ser representada com algumas modificações por meio da Equação 29. Esta forma de apresentação não muda os resultados finais do cálculo do valor da aceleração na direção transversal vertical $\mathrm{a}_{\text {vert }}$ :

$$
a_{\text {vert }}=\left(2 \cdot \pi \cdot f_{1, \text { vert }}\right)^{2} \cdot \frac{\alpha_{i} \cdot P}{k} \cdot \Phi\left[\mathrm{m} / \mathrm{s}^{2}\right]
$$

Na qual:

$\mathrm{f}_{1, \text { vert }}$ : primeira frequência natural da passarela na direção transversal vertical $(\mathrm{Hz})$;

$\alpha_{i} \cdot \mathrm{P} / \mathrm{k}$ : deslocamento estático no centro da passarela devido à amplitude da força dinâmica, que multiplicada por $\left(2 . \pi ._{1, \text { vert }}\right)^{2}$, converte deslocamento em aceleração. Uma alternativa à Equação 28 é obtida pela substituição $\left(2 . \pi . \mathrm{f}_{1} \text {, vert }\right)^{2}=\mathrm{k} / \mathrm{M}$;

k: rigidez no centro do vão $(\mathrm{N} / \mathrm{m}),\left(\mathrm{k}=\mathrm{P} / \mathrm{y}_{\mathrm{est}}\right)$;
P: peso do pedestre igual a $735 \mathrm{~N}$;

$\alpha_{i}$ : coeficiente de Fourier, Tabela 5;

$\Phi$ : fator de amplificação dinâmico obtido graficamente em Rainer, Pernica, Allen (1988), conforme mostrado na Figura 8.

Outra expressão para cálculo do valor da aceleração na direção transversal vertical foi proposta por Rainer, Pernica, Allen (1988), Equação 30, obtida modelando-se a passarela como um sistema de um grau de liberdade. O fator dinâmico de carga $\alpha_{i}$ é escolhido de acordo com o harmônico de carga do pedestre empregado para excitar o modo de vibração da passarela. Este modo de vibração é utilizado para modular a amplitude da excitação, visando simular o efeito de carga móvel. Neste caso, a carga móvel é reduzida a uma carga 
estacionária de amplitude variável. O número de ciclos de vibração também é levado em conta na expressão final do cálculo do valor da aceleração dada pela Equação 30:

$$
\mathrm{a}_{\mathrm{vert}}=\Phi \cdot \frac{2 \cdot \alpha_{\mathrm{i}} \cdot \mathrm{P}}{\mathrm{M}}\left[\mathrm{m} / \mathrm{s}^{2}\right]
$$

Na qual:

M: massa total da passarela $(\mathrm{kg})$;

$\Phi$ : fator de amplificação dinâmico obtido graficamente em Rainer, Pernica, Allen (1988), conforme mostrado na Figura 8, em função do número de ciclos por vão (n) que são necessários para atravessar uma passarela em viga simplesmente apoiada e com certa taxa de amortecimento $\zeta$, considerando passos com comprimento igual a 0,90 $\mathrm{m}$;

P: peso do pedestre igual a $735 \mathrm{~N}$;

$\alpha_{i}$ : coeficiente de Fourier para o ith harmônico, Tabela 5 .

A vantagem dessa formulação é a flexibilidade de escolha do coeficiente de Fourier ai, adotado de acordo com o harmônico da carga de pedestre que excitará a estrutura em sua frequência natural. Por exemplo, uma passarela de frequência natural igual a $4 \mathrm{~Hz}$ pode ser excitada pelo segundo harmônico da carga produzida por um pedestre que se desloca com uma frequência de passo igual a $2 \mathrm{~Hz}$. Entretanto, essa formulação foi concebida para uso apenas em passarelas simplesmente apoiadas em vão simples.

Pimentel e Fernandes (2002) estenderam o procedimento proposto por Rainer, Pernica, Allen (1988), introduzindo o fator de configuração $\left(\mathrm{K}_{\mathrm{PF}}\right)$ que depende do número de vãos, conforme Tabela 4. Este é similar àqueles encontrados na norma britânica BS 5400 (1978) e norma europeia EUROCODE 5 (1993). Estes autores também obtiveram o gráfico da Figura 9, que representa a ampliação do gráfico mostrado na Figura 8, com a extensão do número de ciclos por vão (n) e introdução da taxa de amortecimento $\zeta$ igual a 0,008 , referenciada pela norma britânica BS 5400 (1978). Segundo o próprio Pimentel (1997), amparado pela norma europeia EUROCODE 5 (1993), deve-se eliminar a verificação de conforto humano em passarelas cuja frequência natural na direção transversal vertical seja superior a $5 \mathrm{~Hz}$; do contrário, a aceleração na direção transversal vertical pode ser calculada pela Equação 31:

$$
\mathrm{a}_{\text {vert }}=4 \cdot \pi^{2} \cdot \mathrm{f}_{1, \text { vert }}^{2} \cdot \mathrm{y}_{\text {est }} \cdot \alpha_{\mathrm{i}} \cdot \mathrm{K}_{\mathrm{PF}} \cdot \Phi_{\mathrm{d}}\left[\mathrm{m} / \mathrm{s}^{2}\right]
$$

Na qual:

$\mathrm{f}_{1, \text { vert }}$ : primeira frequência natural da passarela na direção transversal vertical $(\mathrm{Hz})$;

$\mathrm{y}_{\text {est }}$ : flecha devida ao carregamento estático no

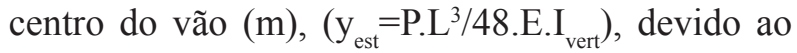
peso do pedestre $(\mathrm{P})$ igual a $700 \mathrm{~N}$;

$\alpha_{i}$ : coeficiente de Fourier para o ith harmônico, Tabela 5;

$\Phi_{\mathrm{d}}$ : fator de amplificação dinâmico obtido graficamente por Pimentel e Fernandes (2002), similar ao obtido por Rainer, Pernica, Allen (1988), conforme Figura 9. Este é obtido a partir do número de ciclos por vão $\left(\mathrm{n}=\mathrm{n}^{\mathrm{o}} \mathrm{i}\right.$ th harmônico.L/ $1_{p}$ ) e do comprimento de passo $\left(1_{p}\right)$ dado na Tabela 3 , necessário para os pedestres cruzarem uma passarela simplesmente apoiada com taxa de amortecimento $\zeta$;

$\mathrm{K}_{\mathrm{PF}}$ : fator de configuração obtido por Pimentel e Fernandes (2002) para passarelas simplesmente apoiadas em vigas simples e contínuas, conforme mostrado na Tabela 4. 
Tabela 4: Fator de configuração KPF

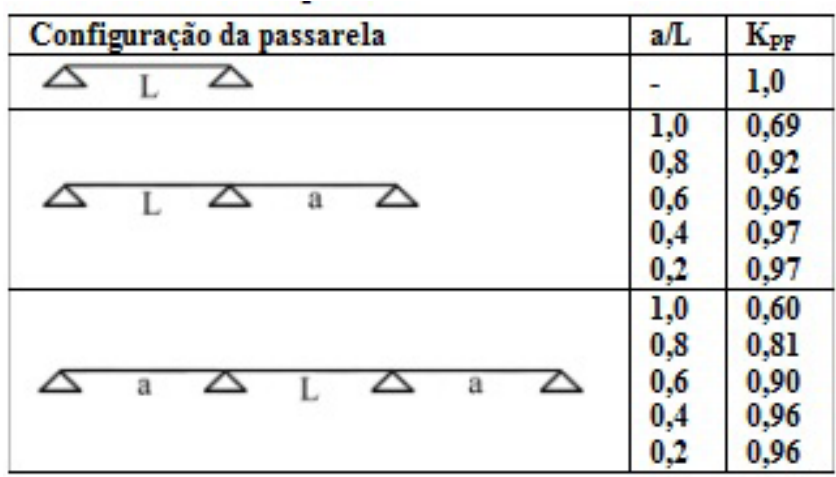

Figura 9: Fator de amplificação dinâmica de uma viga simplesmente apoiada sendo atravessada por uma carga móvel senoidal. Fonte: (Pimentel; Fernandes, 2002)

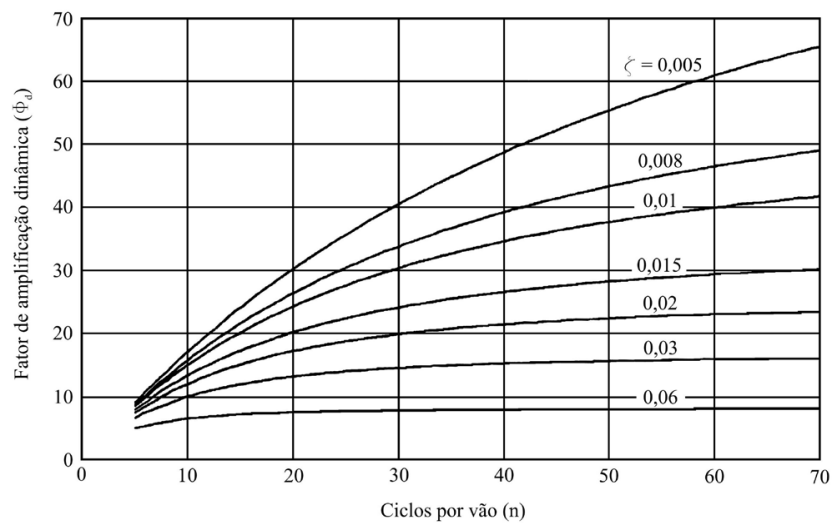

A formulação apresentada por Grundmann, Na qual: Kreunzinger, Schineider (1993) é aplicável a passarelas simplesmente apoiadas de um vão único, baseada na analogia de sistema de um grau de liberdade e frequência natural da passarela igual à frequência de caminhada. $\mathrm{O}$ cálculo do valor da aceleração na direção transversal vertical, produzida por um pedestre cruzando a passarela, é dado pela Equação 32:

$$
\begin{aligned}
& \mathrm{a}_{\text {vert }}=0,6 \cdot \frac{\mathrm{F}}{\mathrm{m}} \cdot \frac{\pi}{\delta}\left(1-\mathrm{e}^{-n \cdot \delta}\right)\left[\mathrm{m} / \mathrm{s}^{2}\right] \\
& \mathrm{F}=\alpha_{\mathrm{i}} \cdot \mathrm{P} \\
& \alpha_{\mathrm{i}}=\mathrm{F}_{\mathrm{i}, \text { vert }} / \mathrm{F}_{0} \\
& \mathrm{~m}=\frac{17}{35} \cdot \mathrm{M}
\end{aligned}
$$

P: peso do pedestre igual a $700 \mathrm{~N}$;

M: massa total da passarela $(\mathrm{kg})$;

$\delta$ : amortecimento crítico em termos de decremento logarítmico $(\delta=2 . \pi . \zeta)$; para a madeira tem-se um valor médio de amortecimento crítico $(\delta=0,075)$ para passarelas de madeira (PETERSEN, 1996);

$n$ : número de passos para cruzar o vão $\left(\mathrm{n}=\mathrm{L} / \mathrm{l}_{\mathrm{p}}\right)$; $1_{\mathrm{p}}$, obtido conforme Tabela 3;

$\mathrm{F}_{0}$ : amplitude da força aplicada no ith harmônico $(\mathrm{N})$;

$\alpha_{i}$ : coeficiente de Fourier para o ith harmônico, conforme Tabela 5. 
Na Equação 29 foram feitas simplificações em sua dedução, visando melhorar a sua aplicação. Inicialmente, um fator dinâmico de carga $\alpha_{1}$ igual a 0,4 foi adotado para o primeiro harmônico da carga que corresponde a um pedestre. Na iniciativa de considerar a validade para altos harmônicos e um pequeno número pedestres, propôs-se o fator de multiplicação S, Figura 10, a ser aplicado na Equação 29, obtido em função da frequência natural da passarela na direção transversal vertical. Por outro lado, o termo $\left(1-\mathrm{e}^{-\mathrm{n} \delta}\right)$ foi obtido da expressão teórica da resposta de um sistema de um grau de liberdade submetido a uma carga senoidal de amplitude constante, (CLOUGH; PENZIEN, 2003). Para considerar o efeito do movimento da carga, foi introduzido um fator multiplicativo igual a 0,6 , que desempenha o papel de converter uma viga simplesmente apoiada no modo fundamental de vibrar correspondente a um sistema de um grau de liberdade.

Figura 10: Fator de multiplicação devido à carga móvel de vários pedestres cruzando a passarela. Fonte: (GRUNDMANN; KREUNZINGER; SCHINEIDER, 1993)

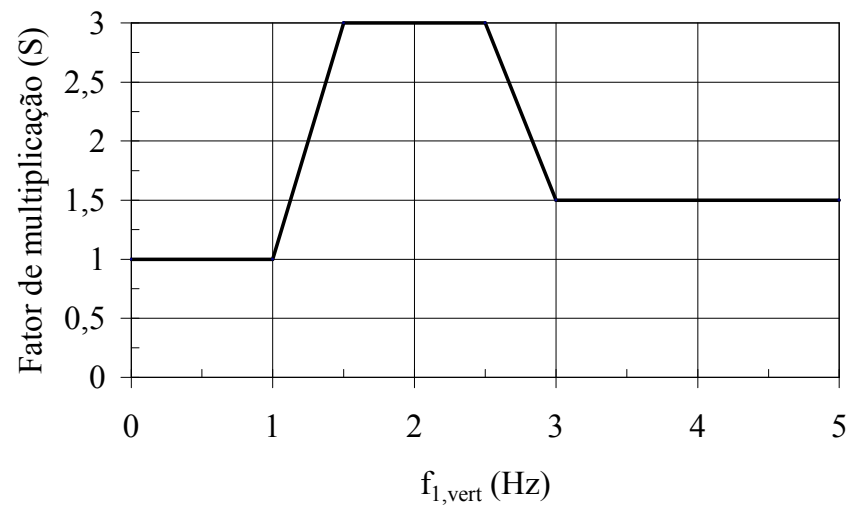

Nas Tabelas 5 e 6, têm-se os fatores dinâmicos de carregamento para o modelo de força de um normal nas direções transversal vertical e transversal horizontal. indivíduo executando atividades de caminhada

Tabela 5: Fatores dinâmicos de carregamento na direção transversal vertical devido ao movimento de caminhada normal (2 Hz). Fonte: (BACHMANN, PRETLOVE, RAINER, 1995)

\begin{tabular}{llllll}
\hline $\mathbf{f p}(\mathbf{2} \mathrm{Hz})$ & $\boldsymbol{\alpha 1 , v e r t}$ & $\boldsymbol{\alpha 2 , v e r t}$ & $\boldsymbol{\alpha 3 , v e r t}$ & $\boldsymbol{\alpha 4 , v e r t}$ & $\boldsymbol{\alpha 5 , v e r t}$ \\
\cline { 2 - 6 } & 0,37 & 0,10 & 0,12 & 0,04 & 0,08 \\
\hline $\boldsymbol{\varphi i}$ & - & $\pi / 2$ & $\pi / 2$ & $\pi / 2$ & $\pi / 2$ \\
$\mathbf{F ~}(\mathbf{H z})$ & $1,4-2,4$ & $2,8-4,8$ & $4,2-7,2$ & $5,6-9,6$ & $7,0-12,0$ \\
\hline
\end{tabular}


Tabela 6: Fatores dinâmicos de carregamento na direção transversal horizontal devido ao movimento de caminhada normal (1 Hz). Fonte: (BACHMANN; AMMAN, 1987)

\begin{tabular}{llllll}
\hline $\mathbf{f p} / \mathbf{2}(\mathbf{1 ~ H z})$ & $\boldsymbol{\alpha 1 , l a t}$ & $\boldsymbol{\alpha 2 , l a t}$ & $\boldsymbol{\alpha 3}$, lat & $\boldsymbol{\alpha 4 , l a t}$ & $\boldsymbol{\alpha 5}$,lat \\
\cline { 2 - 6 } & 0,039 & 0,010 & 0,042 & 0,012 & 0,015 \\
\hline $\boldsymbol{\varphi \mathbf { i }}$ & - & $\pi / 2$ & $\pi / 2$ & $\pi / 2$ & $\pi / 2$ \\
$\mathbf{f}(\mathbf{H z})$ & $0,6-1,1$ & $1,2-2,2$ & $1,8-3,3$ & $2,4-4,4$ & $3,0-5,5$ \\
\hline
\end{tabular}

\section{Considerações finais}

As comparações entre os critérios de vibrações nas direções transversal vertical e transversal horizontal são apresentadas nas Figuras 11 e 12, respectivamente. Os valores de aceleração contidos na ISO 10137 (2005), em RMS, foram convertidos para grandeza (pico da aceleração) similar aos demais critérios normativos, multiplicando os valores da aceleração RMS, mostrados nas Figuras 4 e 5 , pelos fatores $2^{0,5}$ e 60 .

Figura 11: Comparativo entre critérios normativos para aceitação de vibração na direção transversal vertical

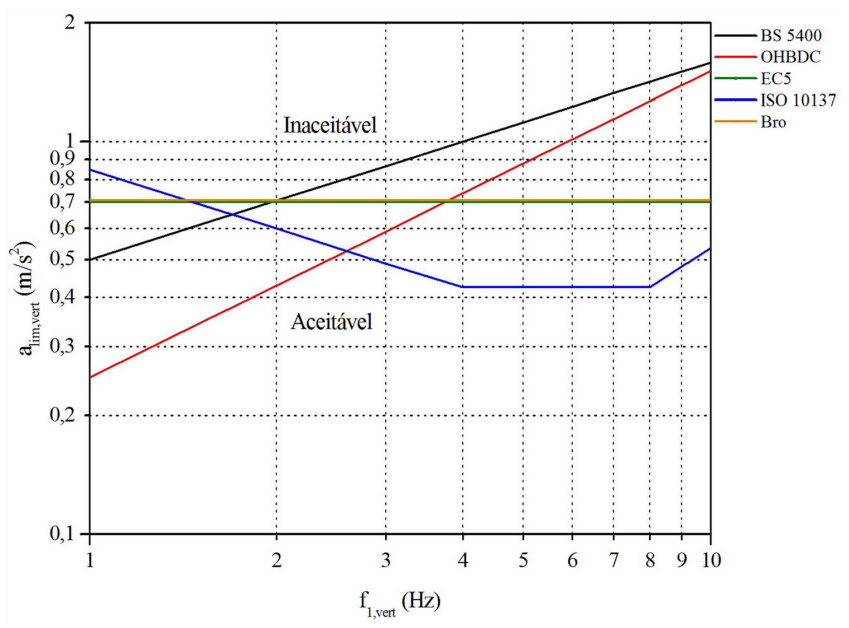

Figura 12: Comparativo entre critérios normativos para aceitação de vibração na direção transversal horizontal.

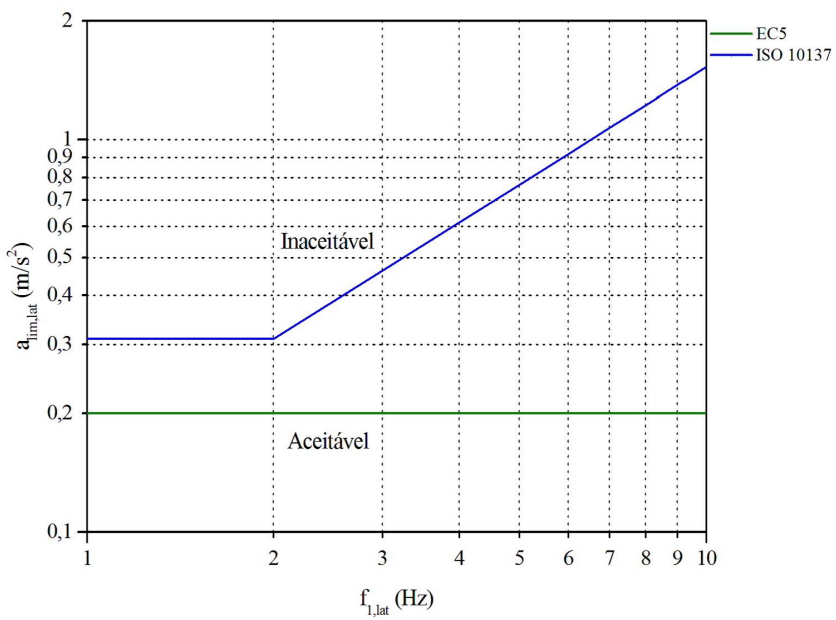


Uma comparação da vibração na direção transversal vertical mostra que EUROCODE 5 (1993, 2004) e BRO (2004) apresentam frequência independente e aceleração limite de $0,7 \mathrm{~m} / \mathrm{s}^{2}$, para uma passarela com frequência natural transversal vertical igual a $2 \mathrm{~Hz}$, que é a principal frequência de caminhada. O critério da BS 5400 (1978) fornece uma aceleração na direção transversal vertical de aproximadamente $0,707 \mathrm{~m} / \mathrm{s}^{2}$ e, na ISO 10137 (2005), encontra-se um valor de aceleração na direção transversal vertical de aproximadamente 0,6 $\mathrm{m} / \mathrm{s}^{2}$, ambas para uma frequência natural na direção transversal vertical igual a $2 \mathrm{~Hz}$.

Uma comparação dos critérios de vibração na direção transversal horizontal mostra que EUROCODE 5 (1993, 2004) apresenta uma aceleração limite de $0,2 \mathrm{~m} / \mathrm{s}^{2}$ independente da frequência. A ISO 10137 (2005) dá uma aceleração de aproximadamente $0,31 \mathrm{~m} / \mathrm{s}^{2}$ até uma frequência de $2 \mathrm{~Hz}$. Tanto a norma BS 5400 (1978), quanto a norma BRO (2004) apresentam um critério de aceleração para vibração na direção transversal horizontal. Contudo, a BS 5400 (1978) condiciona que, se a frequência natural da vibração na direção transversal horizontal é menor do que $1,5 \mathrm{~Hz}$, o projeto deve considerar o risco de movimento na direção transversal horizontal de magnitude inaceitável.

A norma britânica BS 5400 (1978) propõe um modelo de carregamento de pedestres somente na direção transversal vertical e não na direção transversal horizontal. A ISO 10137 (2005) modela os carregamentos, nas direções transversal vertical e transversal horizontal, impostos por um pedestre. Notou-se que, na modelagem do carregamento na direção transversal horizontal do pedestre, é assumido que parcela do peso estático do pedestre age na direção transversal horizontal. O EUROCODE 5 (1993) propõe modelos de carregamentos para as direções transversal vertical e transversal horizontal, somente para o sistema estrutural em vigas retas simplesmente apoiadas. É valido observar que todos os critérios normativos e as indicações de pesquisadores foram estabelecidos e aproximados supondo que os carregamentos de pedestres são periódicos.

O EUROCODE 5 (2004) apresenta uma formulação simplificada em relação ao EUROCODE 5 (1993), tomando apenas o caso de passarelas simplesmente apoiadas, sem levar em consideração o caso de vãos contínuos. Também não apresenta uma formulação indicando uma força a ser aplicada em passarelas de outros tipos de sistemas estruturais, para ser obtido o valor de acelerações nas direções transversal vertical e transversal horizontal. Este código normativo já evoluiu em relação aos demais, pois apresenta uma formulação para o cálculo do valor da aceleração na direção transversal vertical que leva em conta o caso de pessoas correndo sobre a passarela de madeira.

Os procedimentos de cálculo das acelerações do EUROCODE 5 (2004), versão atual da norma europeia, são baseados no desenvolvimento do trabalho de Grundmann, Kreunzinger, Schineider (1993), podendo-se dizer que as Equações 23 a 27 são uma versão simplificada desses autores. A primeira parte da Equação 23 refere-se ao primeiro harmônico de caminhada e recomenda o cálculo da aceleração na direção transversal vertical, para a frequência natural da passarela menor ou igual a 2,5 Hz. A segunda parte da Equação 23 refere-se ao cálculo da aceleração, para o segundo harmônico de caminhada com frequência natural maior do que $2,5 \mathrm{~Hz}$ e menor ou igual a 5,0 Hz. A Equação 24 leva em conta o primeiro harmônico com frequência maior do que $0,5 \mathrm{~Hz}$ e menor ou igual a $2,5 \mathrm{~Hz}$ na direção transversal horizontal. A Equação 25 leva em conta o primeiro harmônico para o movimento de corrida na direção transversal vertical.

A proposta de Rainer, Pernica, Allen (1988) permite trabalhar com todos os harmônicos da ação dos pedestres, segundo Pimentel (1997) e Pretlove, Rainer(1995).Aspropostas doEUROCODE5(1993, 2004) e de Grundmann, Kreunzinger, Schineider (1993) levam em conta o efeito de ressonância, por 
meio de um coeficiente de amplificação dinâmica que depende da frequência natural da passarela. Os autores citados em seus trabalhos levantam o problema da sincronização de multidão de pedestres que estejam cruzando a passarela e apresentam procedimento para avaliação do problema. Também apresentam os procedimentos de dimensionamento da passarela em função das vibrações laterais.

Do estudo realizado por Pimentel e Fernandes (2002), que diz respeito à investigação da aplicabilidade de formulações simplificadas propostas na literatura, na análise de vibrações produzidas por pedestres em passarelas, concluiuse que, nos vários casos examinados, a formulação proposta apresentou estimativas mais precisas para as acelerações.

O critério de dimensionamento da ABNT NBR 7190 (1997) não apresenta nenhuma indicação relativa aos limites de frequência e aceleração para as passarelas de madeira. A indicação da ABNT NBR 7190 (1997) é relativa a pisos de residências e escritórios; caso fosse estendida às passarelas, com certeza levaria à construção de estruturas robustas, acarretando uma solução antieconômica, uma vez que o critério estabelece uma frequência superior a $8 \mathrm{~Hz}$. Segundo a ABNT NBR 7190 (1997), este critério é atendido caso os pisos tenham uma flecha imediata inferior a $15 \mathrm{~mm}$, referente a um carregamento de curta duração e seja utilizado, na formulação, o módulo de elasticidade efetivo Ec0,ef.

A sugestão da ABNT NBR 7190 (1997), de uma frequência superior a $8 \mathrm{~Hz}$, é uma primeira maneira de evitar problemas de vibrações, fazendo com que a estrutura tenha uma primeira frequência natural pelo menos acima do alcance do terceiro harmônico, ou seja, maior que três vezes a frequência de caminhada $(3 \cdot 2,4=7,2 \mathrm{~Hz})$. Por isso, pisos com a primeira frequência natural acima de $8 \mathrm{~Hz}$ estariam salvaguardados desse efeito de ressonância. Na realidade, para estruturas com amortecimento relativamente alto, será suficiente que a primeira frequência natural seja acima de $5 \mathrm{~Hz}$, isto é, a contribuição do terceiro harmônico já não será tão relevante.

Tomando como base os estudos realizados nos vários critérios normativos referenciados no texto deste artigo, sugere-se o EUROCODE 5 (2004) como critério a ser adotado para verificação das vibrações excessivas em passarelas de madeira no Brasil, principalmente no caso particular de passarelas simplesmente apoiadas, a situação mais frequente, tanto na zona urbana, quanto rural.

\section{Agradecimentos}

Ao CNPq, Conselho Nacional de Desenvolvimento Científico e Tecnológico - Brasil, e à CAPES, Coordenação de Aperfeiçoamento de Pessoal de Nível Superior - Brasil, pelo apoio concedido a esta pesquisa na forma de bolsa (Doutorado) e taxa de bancada.

\section{Referências}

AMERICAN ASSOCIATION OF STATE, HIGHWAY AND TRANSPORTATION OFFICIALS. AASHTO. Guide specifications for design of pedestrian bridges. Washington D. C., Aug. 1997.

ASSOCIAÇÃO BRASILEIRA DE NORMAS TÉCNICAS - ABNT. NBR 7188: Carga móvel em ponte rodoviária e passarela de pedestre. Rio de Janeiro, 1982.

NBR 7190: projeto de estruturas de madeira. Rio de Janeiro, 1997.

BACHMANN, H.; AMMANN, W. Vibrations in structures induced by man and machines: structural engineering document 3E. Zürich: IABSE, 1987.

BACHMANN, H.; PRETLOVE, A. J.; RAINER, H. Dynamic forces from rhythmical human body motions. In: BACHMANN, $H$. et al. Vibration problems in structures: practical guidelines. Berlin: Birkhauser, 1995. p. $185-187$.

BLANCHARD, J.; DAVIES, B. L.; SMITH, J. W. Design criteria and analysis for dynamic loading of footbridges: Symposium on dynamic behaviour of bridges. Crowthorne: Ministry of Transport, Department of the Environment, Transport and Road Research Laboratory 
(TRRL). Supplementary Report, v. 275, p. 90-106, 1977. BRITISH STANDARDS INSTITUTION. BS5400: Steel, concrete and composite bridges: specification for loads: Part 2, Appendix C. London: British Standards Association, 1978.

BRO 2004. Publikation 2004:56: Vägverkets allmänna tekniska beskrivning för nybyggande och förbättring av broar: Svensk Byggtjänst. Stockholm: Sverige, 2004.

CLOUGH, R. W.; PENZIEN, J. Dynamics of structures. 3th ed. Berkeley: CSi, 2003.

COMITÉ EURO-INTERNATIONAL DU BÉTON. CEB. Vibration problems in structures: Practical guidelines. London: Thomas Telford Services, Aug. 1993. (Bulletin d'Information 209).

DALLARD, P.; FITZPATRICK, A. J.; FLINT, A.; LOW, A.; RIDSDILL SMITH, R.; WILLFORD, M.; ROCHE, M. London millennium bridge: Pedestrian-induced transversal horizontal vibration. Journal of Bridge Engineering, Reston, v. 6, n. 6, p. 412-417, Nov./Dec. 2001.

EUROPEAN COMMITTEE FOR STANDARDIZATION. EUROCODE 5 (prEN 19951-2): design of timber structures, Part 1-2: Bridges. Brussels, 1993.

. EUROCODE 5 (prEN 1995-1-2): design of timber structures. Part 1-2: Bridges. London, 2004.

EYRE, R.; CULLINGTON, D. W. Human tolerance levels for bridge vibration. Crowthorne: Ministry of Transport, Department of the Environment, Transport and Road Research Laboratory (TRRL). Research Report, n. 18, 1985.

GRUNDMANN, H.; KREUNZINGER, H.; SCHNEIDER, M. Dynamic calculations of footbridges. Bauingenieur, Berlin, v. 68, n. 5, p. 215-225, 1993.

INTERNATIONAL ORGANIZATION FOR STANDARDIZATION. ISO 10137: bases for design of structures: serviceability of buildings and pedestrian walkways against vibration. Geneva, 2005.

ISO 2631-2: evaluation of human exposure to whole-body vibration, Part 2, Continuous and shockinduced vibration in buildings (1 to $80 \mathrm{~Hz}$ ). Geneva, 1989.

ONTARIO HIGHWAY BRIDGE DESIGN CODE OHBDC. Ministry of transportation and communications: highway engineering division, 3th ed. Ontario, 1991.

PETERSEN, C. Dynamik der Baukonstruktion. Berlin: Vieweg, 1996.
PIMENTEL, R. L. Vibrational performance of pedestrian bridges due to human-induced loads. 1997. Thesis (PhD) - University of Sheffield, Sheffield.

PIMENTEL, R. L.; FERNANDES, H. M. B. A simplified formulation for vibration serviceability of footbridges. In: INTERNATIONAL CONFERENCE ON DESIGN AND DYNAMIC BEHAVIOUR OF FOOTBRIDGES: Footbridge 2002. 1., 2002, Paris. Proceedings... Paris: CD-ROM, p. 20-22. Nov. 2002.

PIMENTEL, R. L.; PAVIC, A.; WALDRON, P. Evaluation of design requirements for footbridges excited by transversal vertical forces from walking. Canadian Journal of Civil Engineering, Vancouver, v. 28, n. 5, p. 769-777, 2001.

PRETLOVE, A. J.; RAINER, J. H. Human response to vibrations. In: BACHMANN, $\mathrm{H}$. et al. Vibration problems in structures: practical guidelines. Basel: Birkhauser, 1995.

RAINER, J. H.; PERNICA, G.; ALLEN, D. E. Dynamic loading and response of footbridges. Canadian Journal of Civil Engineering, Vancouver, v. 15, n. 1, p. 66-71, 1988.

SEGUNDINHO, P. G. A. Estudo das vibrações induzidas por pedestres em passarelas de madeira. 2010. Tese (Doutorado em Engenharia de Estruturas) - Escola de Engenharia de São Carlos - USP, São Carlos.

SMITH, J. W. Vibrations in structures: applications in civil engineering design. London: Chapman and Hall, 1988.

SWISS SOCIETY OF ENGINEERS AND ARCHITECTS. SIA 160: Action on structures, Zürich, 1989.

WRIGHT, D. T.; GREEN, R. Human sensitivity to vibration: report. Kingston. Ontario, 1959. (Queen's University, n. 7).

ZIVANOVIC, S.; PAVIC, A.; REYNOLDS, P. Vibration serviceability of footbridges under human-induced excitation: a literature review. Journal of Sound and Vibration, Southampton, v. 279, p. 1-74, 2005.

Recebido em 4 Agosto, 2010 - Received on August 4, 2010.

Aceito em 3 Fevereiro, 2011 - Accepted on February 3, 2011. 
